\title{
APPLICATION OF FUZZY LOGIC APPROACH TO STRUCTURE BASED COMPARISON OF IN-PARALLEL PLANAR MANIPULATORS
}

\author{
A. B. Srinivasa Rao \\ Aditya Engineering College, Surampalem-533437, E.G. Dt. AP \\ Corresponding author: annamsrinu@yahoo.com \\ Received 14 June, 2009; Revised 18 March, 2010
}

\begin{abstract}
Planar In-parallel Manipulations, which are nothing but multi degree-of-freedom closed kinematic chains can be modelled as fuzzy systems, so that the fuzzy logic can be applied. It is shown that the fuzzy entropy can be utilized to compare numerous distinct chains, with the same number of links and degrees of freedom from the view point of parallelism so that the designer can have an idea, at the conceptual stage of design regarding suitability of chains, from the view point of rigidity, work space and accumulation of joint errors. Spatial chains are also dealt with. Aspect of work space is verified experimentally.
\end{abstract}

Keywords : Fuzzy Vector, Fuzzy Entropy, Degree-of-freedom

\section{INTRODUCTION}

Basically the mechanical structure of a robot is composed of cantilever beams forming a sequence of arm links connected by hinged joints. Such robot arm is usually referred to as serial type which has inherently poor mechanical stiffness and accuracy and hence not suitable for heavy duty applications. Further it suffers the disadvantage of accumulation of joint errors from shoulder to the end effector. Multi-degree-of-freedom (d.o.f) closed kinematic chains are now receiving active consideration for application as in parallel or platform type robots $(1,2,3)$. Though the problems of direct kinematics is more difficult the in-parallel type robots are inherently more rigid and hence they are seen as potential substitutes for heavy duty applications.

A number of distinct planar kinematic chains with the same number of links and degree -offreedom are available to the designer. Hence the designer must be able to compare all these chains and select the best. It is necessary to grade all the distinct multi d.o.f. kinematic chains for parallelism since they will reveal characteristics like work space, rigidity and accumulation of joint errors. An attempt is made in this paper to deal with this aspect using fuzzy logic.

Taking the probabilistic fuzzy logic into account the author made an attempt to make a comparison between distinct multi d.o.f. kinematic chains for parallelism by considering every link in the chain as a fuzzy number. In linkages displacements or velocities depend both upon the structure and dimensions. Each chain depending upon the mobility exhibits a certain character. If two distinct chains with the same link assortments and link dimensions are considered, the link with greater mobility is expected to have greater velocities. Identical 
changes of link dimensions in both the chains do not alter this character. in order to know the mobility of links in a chain, which is an important item to be considered for measure of parallelism and Noting that the fully connected chain is least mobile, author decided to use the concept of "entropy" specified in fuzzy theory, which is a measure of uncertainty for deciding the parallelism of a chain. It can be generalized that links of a chain with greater entropy are more mobile i.e. the displacements and velocities can be higher.

The fuzzy logic is extended to spatial chains also, having joints with one or more degrees of freedom such as revolute and spherical etc., also, so that all the platform-type robot structures can be compared form the point of accumulation of joint errors.

\section{FUZZY THEORY}

The principles of fuzzy theory are discussed here to the extent necessary (4). Mostly we are used to two-state logic, symbolized through the binary digits (bits) 0 and 1. But another kind of logic is possible, based on a multiplicity of possible logic states represented by the decimal values 0 , $0.1,0.2, \ldots 1.0$ The system that deals with these multiples is called fuzzy logic (4). Neural networks and fuzzy systems have one thing in common i.e., both resemble our instinctive thought patterns. Fuzzy logic contains concepts that correlate with the gray-area terms that people use - "Sort of", "Mostly", "very" and "not much". The fuzzy index on a scale of 0 to 1 is a measure of membership in a set. It is important to recognize that fuzzy index is different from the probability, the resemblance, if any, is only superficial. Like probability, fuzzy membership is also defined on a scale from 0 to 1 . Further, zero and unity in each of the systems convey similar meaning. A probability of 1 represents certainty of occurrence of an event and a fuzziness of 1 represents certainty that an entity belongs to a particular set.

Consider the class of stormy nights (4). It is not necessarily obvious whether a particular night belongs to this class. If on a particular night, a strong warm wind is blowing, membership of that night in the class of stormy nights might be designated as 0.3 . On the other hand if there is a strong wind blowing at about 15 knots accompanied by rain, the night may have a membership of 0.7 in the class of stormy nights. The numerical values of the membership in fuzzy-logic are called "fits" analogous to "bits" in binary logic. A fuzzy vector is a list such as

$$
\mathrm{A}=\begin{array}{lllll}
0 & 0.3 & 0.7 & 0.4 & 0.8
\end{array}
$$

\section{Crisp Vector:}

Considering once again the above example of stormy nights, a pleasant spring nights may be assigned a membership of zero in the class of stormy nights and a gale with pelting rain and lighting can be assigned a membership of 1 . In both the cases, the fuzzy (uncertain) variables has become crisp. Hence, a crisp vector (C) consists of 0 and 1 only.

Eg., $\quad \mathrm{C}=0 \begin{array}{lllll}0 & 1 & 0 & 1 & 1\end{array}$

Nearest crisp vector, $\mathrm{C}_{\mathrm{n}}$ is a vector in which all its "fits" are either 0 or 1 . If the value of the "fit", $\mathrm{f}$ in the vector $\mathrm{A}$ is $0 \leq \mathrm{f} \leq 0.5$, the corresponding "fit" in the nearest crisp vector is ' 0 ' and the value of the fit will be 1 if $\mathrm{f}>0.5$. Similarly, farthest crisp vector $\mathrm{C}$ is a vector in which 
all the fits are either 0 or 1 . The fit in this vector $\mathrm{C}$ assumes a value 1 if $\mathrm{f}$ in the vector $\mathrm{A}$ has value $0 \leq \mathrm{f} \leq 0.5$, otherwise 0 . For example, for the fuzzy vector.

$\mathrm{A}=0 \quad 0.3 \quad 0.7 \quad 0.4 \quad 0.8 \quad$ the nearest crisp vector $\mathrm{C}_{\mathrm{n}}$ will be $\mathrm{C}_{\mathrm{n}}=0,0,1,0,1$ and the farthest crisp vector

$\mathrm{C}_{\mathrm{f}}=1 \quad 1 \quad 0 \quad 1 \quad 1 \quad 0$

\section{Distance}

Distance between a fuzzy vector and the corresponding crisp vector is defined as the sum of the adjustments that are necessary to make A equal to the crisp vector. For example nearest distance is 1.2 .

\section{Pathological Vector}

Pathological vector is one in which all fits are 0.5. It represents the fuzziest (or least certain) possible vector in its $n$-dimensional space.

\section{Fuzzy Entropy}

Fuzzy entropy $(\Phi)$ is a measure of uncertainty and is defined for any fuzzy vector A as the ratio of its distance from the nearest crisp vector to that from the farthest vector. For example, for the fuzzy vector $\mathrm{A}=\begin{array}{lllll}0 & 0.3 & 0.7 & 0.4 & 0.8\end{array}$ the distance from the nearest crisp vector is 1.2 while it is 3.8 from the farthest crisp vector. Hence fuzzy entropy $\Phi=0.316$

\section{ADAPTION}

It is known that a serial manipulator will have greater work space, less rigidity and greater positional error of the end-effector due to joint errors compared to the in-parallel manipulators with the same degree-of-freedom. A planar 3 d.o.f. serial manipulator is shown in figure 1a and an in-parallel 8-link planar manipulator with the same degree-of-freedom is shown in figure 1 (b).

In the following, an attempt is made to develop a quantitative measure using fuzzy logic to establish the degree-of-parallelism or serialism.

In order to adapt fuzzy-logic and to be consistent with actual conditions the following observations are made for planar structures viewing them as a combination of links and joints.

In a closed kinematic chain, motion introduced at one joint is transmitted to at least two other joints. Sharing of motion or power is considered as parallelism. A joint from which motion is transferred only to two other joints is shown at "A" in figure1 (a\&b), on the other hand when the motion received by a joint is transferred simultaneously to more than two joints the joint is considered to be more parallel. Such a joint is shown at B in figure 1 (b). A least parallel or most serial joint is formed by two binary links only, while a more parallel joint can be formed by joining (i). A binary link to a ternary link (ii). A ternary link with a ternary link etc., Each binary link is considered to have one design parameter. Thus a least parallel or most serial-joint involves only two design parameters and hence it can numerically be represented by $1 / 2=0.5$. 


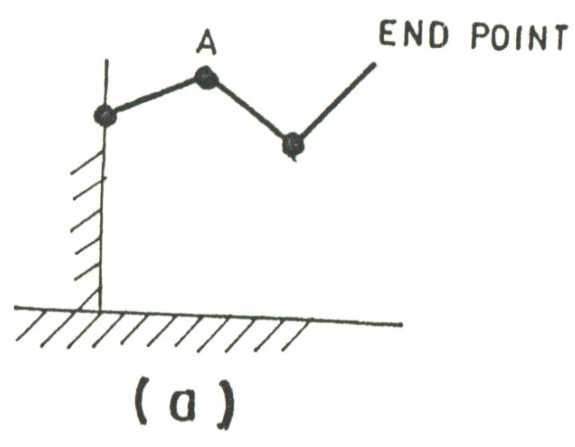

SERIAL MANIPULATOR

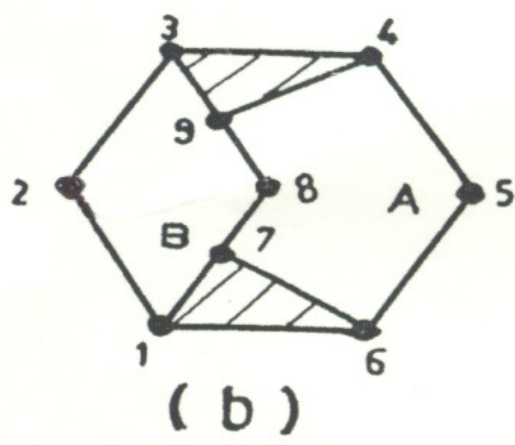

IN-PARALLEL MANIPULATOR

\section{FIGURE 1}

Now consider a joint, such as B, in figure1 (b). This joint is directly connected to three other joints i.e., motion or power introduced at joint $B$ will be shared by three other joints and thus it can be considered as less serial compared to joint A figure 1 (a) and is numerically represented by $1 / 3=0.33$. In kinematic chains, joints which are connected directly to four or more joints may exist and such joints can be considered as less serial than the joint $\mathrm{B}$ - explained above and a numerical value of $1 / 4$ or $1 / 5$ can be assigned depending upon the number of other joints $(\mathrm{J})$ to which it is directly connected. In other words, quantitative measure $1 / \mathrm{j}$ can be used to know how serial a joint is, highest value of a serial joint being $1 / 2$. Thus every joint in a chain can be assigned a fuzzy membership or fit value of $1 / \mathrm{J}$.

Each joint signifies parallelism (or less serialism); is definite and fuzziness comes only in assigning fit value to a joint to quantify serialism.

Another reason that supports the assignment of $1 / \mathrm{J}$ for a joint is that a serial joint such as $\mathrm{A}$ figure $1(\mathrm{a} \& \mathrm{~b})$ will get a assigned fuzzy membership of 0.5 . This enables representation of every serial manipulator of

n-degree-of-freedom by a n-dimensional pathological fuzzy vector e.g., for the chain, shown in figure 1 a fuzzy vector (which includes all the joints of a chain) becomes a three dimensional pathological vector,

$\begin{array}{lll}0.5 & 0.5 & 0.5\end{array}$

The fuzzy -entropy, of such a vector is 1.0 (maximum value an entropy can assume). The fact that a truly serial manipulator of any dimensions or any number of joints will have the entropy 1.0, all manipulators which are less serial or more parallel must have an entropy value less than 1.0. This fact is illustrated below.

Let us compare the in-parallel chain, figure $1 \mathrm{~b}$ with the serial type figure 1(a). Each joint in a chain is to be assigned a membership number consistent with 0.5 assigned earlier to a binary joint and also with the possible accuracy (certainty). As explained earlier, the fuzzy membership 
of a binary joint is taken inversely proportional to the number of design parameters taking part in the joint. Similarly, a ternary joint i.e., a joint which is connected to three other joints e.g., point 8 in figure 1(b) can be considered to have three design parameters participating in the joint. In general a fuzzy membership number which is related inversely to the number of design parameters (or joint-connectivity) can be assigned to a joint. Thus, a ternary joint will have a fuzzy membership 0.33. In this work no attempt is made to rewrite the membership as 0.3 to fall in line with the fuzzy theory though all the general principles of fuzzy theory are retained. With the above understanding all the joints 1,2 etc., of the closed kinematic chain, figure1(b) can be represented by a fuzzy vector

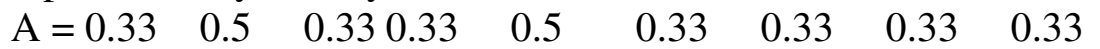

Its nearest crisp vector is $\mathrm{C}_{\mathrm{n}}=\begin{array}{llllllllll}0 & 0 & 0 & 0 & 0 & 0 & 0 & 0 & 0 \text {, Hence nearest distance }=3.31\end{array}$

Farthest crisp vector is $\mathrm{C}_{\mathrm{f}}=\begin{array}{lllllllll}1 & 1 & 1 & 1 & 1 & 1 & 1 & 1 & 1\end{array}$

Hence, farthest distance $=5.69$

Therefore, Fuzzy Entropy $=3.31 / 5.69=0.5817$

It may be noticed that the fuzzy entropy (5.82) of the closed chain, figure 1(b) with three-degreeof freedom is lower than the entropy (1.0) of the serial type, figure 1 (a) with the same degree-offreedom. Thus, the magnitude of the fuzzy entropy can be taken as the criterion to judge parallelism of a chain i.e., "lesser the entropy greater is the parallelism or less serial".

\section{COMPARISON OF IN-PARALLEL MAINPULATORS}

After establishing the entropy as a measure of parallelism it is necessary to compare, among themselves, all the distinct chains with the same number of links and degree-of-freedom from the view point of parallelism.

In order to compute the fuzzy entropy for a closed kinematic chain with a given number of links and d.o.f. It becomes necessary to write a fuzzy-vector representing the chain. Closed kinematic chains differ on two counts (i) Difference in type of joints and their number and (ii) Influence of the topology. The later aspect need be considered only in the chains for which the number and type of joints are the same. In what follows, both the aspects are considered and illustrated.

\section{CHAINS WITH DIFFERENT TYPES OF JOINTS}

Consider two seven link 2 d.o.f. chains, figure 2 (a\&b). Joints are numbered 1,2,3 etc. It may be seen that both the chains contain joints different in nature e.g., in the chain figure $2 b$ two ternary links are directly joined (joint-8) and no such joint exists in the chain figure 2a. Recalling that a joint is assigned a fuzzy membership number inversely proportional to the number of other joints to which it is directly connected (here after called joint connectivity) the fuzzy-vectors for different chains can be written. For the chain in figure 2 (a) the fuzzy vector is 

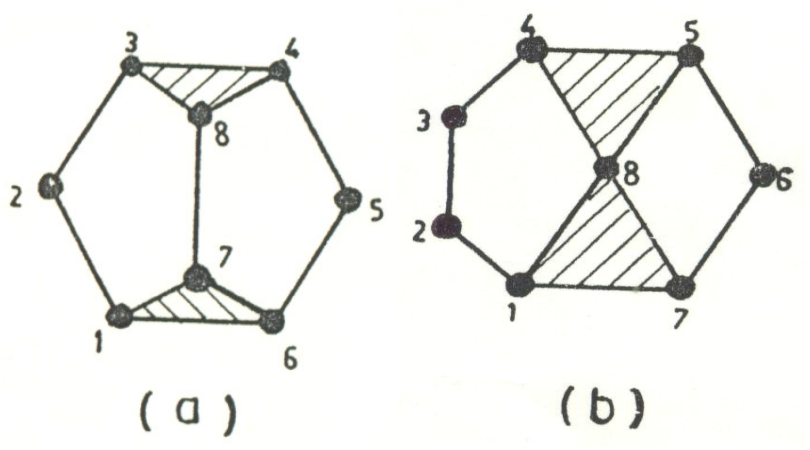

SEVEN LINK 2 d.o.f CHAIN

FIGURE 2

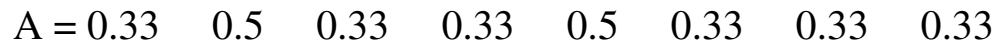

Hence, nearest crisp vector is $\mathrm{C}_{\mathrm{n}}=\begin{array}{lllllllll}0 & 0 & 0 & 0 & 0 & 0 & 0 & 0\end{array}$

Therefore, nearest distance $=2.98$.

Farthest crisp vector is $\mathrm{C}_{\mathrm{f}}=1 \quad 1 \quad \begin{array}{lllllllll}1 & 1 & 1 & 1 & 1 & 1 & 1\end{array}$

Therefore, farthest distance $=5.02$

And Fuzzy Entropy $=2.98 / 5.02=0.594$

For the chain, figure 2(b) Fuzzy vector is

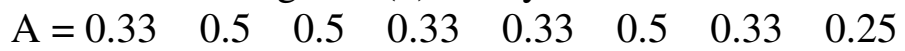

The nearest crisp vector is $\mathrm{C}_{\mathrm{n}}=\begin{array}{llllllll}0 & 0 & 0 & 0 & 0 & 0 & 0 & 0\end{array}$

Therefore, nearest distance is $=3.07$

The farthest crisp vector is $\mathrm{C}_{\mathrm{f}}=\begin{array}{llllllll}1 & 1 & 1 & 1 & 1 & 1 & 1 & 1\end{array}$

Therefore, farthest distance is 4.93 , and fuzzy entropy $=3.07 / 4.93=0.622$

Comparison of fuzzy entropies reveals that the entropy is more in case of the chain, figure2 (b) which indicates that this chain is more "serial" compared to that of the chain, figure 2(a).

The above example leads to a conclusion that in general, the chains consisting of joints with higher connectivity will have greater fuzzy entropy and hence tend to be more "serial".

\section{INFLUENCE OF TOPOLOGY}

Chains in which the type of joints are different can be compared easily as explained in the foregoing section. Nevertheless, there are chains with the same type and number of joints but with different chain topology e.g., the two seven link chains with 2 d.o.f; figure 3 (a\&b). The joints are numbered 1,2,3 etc., in both the chains. Since both the chains contain the same number and type of joints their fuzzy vectors and hence the fuzzy entropies will be the same. Thus no comparison can be made on the lines suggested in the earlier section. For dealing with such chains fuzzy adjacency matrices, $\mathrm{F}=\left(\mathrm{f}_{\mathrm{ij}}\right)$ are proposed. In these matrices the element $\mathrm{F}_{\mathrm{ij}}$ assumes the following values

$\mathrm{F}_{\mathrm{ij}}=0$ if the joints $\mathrm{i}$ and $\mathrm{J}$ are not directly joined

$F_{i j}=$ fuzzy numerical value assigned to joint ' $J$ ' if it is directly connected to joint ' $\mathrm{i}$ ' 


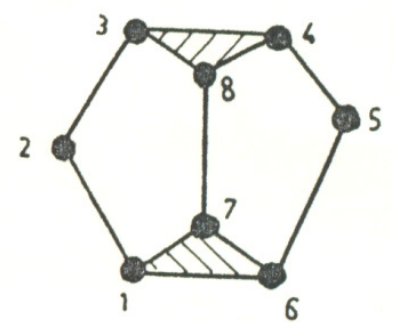

(a)

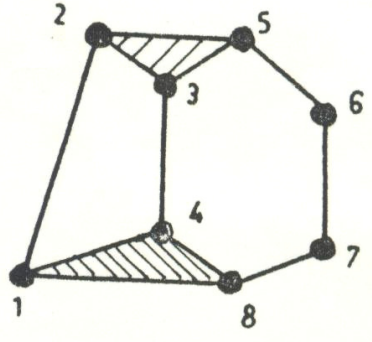

( b )

SEVEN LINK 2 d.o.f CHAIN

FIGURE 3

For the chain in figure 3 (a)

$$
\mathrm{F}=\left(\begin{array}{llllllllll}
0.33 & 0.5 & 0 & 0 & 0 & 0.33 & 0.33 & 0 \\
0.33 & 0.5 & 0.33 & 0 & 0 & 0 & 0 & 0 \\
0 & 0.5 & 0.33 & 0.33 & 0 & 0 & 0 & 0.33 \\
0 & 0 & 0.33 & 0.33 & 0.5 & 0 & 0 & 0.33 \\
0 & 0 & 0 & 0.33 & 0.5 & 0.33 & 0 & 0 \\
0.33 & 0 & 0 & 0 & 0.5 & 0.33 & 0.33 & 0 \\
0.33 & 0 & 0 & 0 & 0 & 0.33 & 0.33 & 0.33 \\
0 & 0 & 0.33 & 0.33 & 0 & 0 & 0.33 & 0.33
\end{array}\right)
$$

The elements in the $i^{\text {th }}$ row can be considered as the fuzzy vector $A_{i}$ of $i^{\text {th }}$ joint and the corresponding fuzzy entropy can be evaluated. The sum of the fuzzy entropies of n-joints can then be taken as the adjacency entropy of the chain. For example, for joint 1 , the fuzzy vector $\mathrm{A}_{1}$ is

$$
\begin{array}{llllllll}
0.33 & 0.5 & 0 & 0 & 0 & 0.33 & 0.33 & 0
\end{array}
$$

Which will lead to an entropy of $1.49 / 6.51=0.23$ Fuzzy vector of the joint 2 is

$$
\begin{array}{llllllll}
0.33 & 0.5 & 0.33 & 0 & 0 & 0 & 0 & 0
\end{array}
$$

And the corresponding entropy is $1.16 / 6.84=0.17$

Fuzzy vector of joint 7 is $\quad \begin{array}{lllllllll}0.33 & 0 & 0 & 0 & 0 & 0.33 & 0.33 & 0.33 & \text { and the corresponding entropy }\end{array}$ is $1.32 / 6.68=0.198$

Computing entropy for all the joints we get the adjacency fuzzy entropy of the chain $=1.656$

The fact that the entropy is greater than 1.0 should not had to confusion as the entropy computed above pertains to eight fuzzy vectors. 
Similarly the adjacency matrix, for the chain in figure 3(b) can be written and its fuzzy entropy can be computed. Adjacency -Fuzzy entropy for the chain, in figure 3(b) is 1.651. Comparison of the fuzzy entropies for the chains figure 3(a\&b) shows that the chain figure 3(a) has greater entropy and hence it is more "serial"

\section{APPLICATION TO SPATIAL CHAINS}

Spatial chains may consist of joints like revolute, cylindrical, spherical etc. Chains, to be compared, need be represented by fuzzy vectors. Each joint may be assigned to a group represented by numbers $0.2,0.3$ etc., depending upon the joints possible contribution to motion transmission. The degree-of-freedom (d.o.f) of a joint influences the motion transmission. With this understanding, revolute, cylindrical, spherical joints etc., can be assigned numbers $0.1,0.2,0.3$ etc., respectively on $0-1$ scale since, the d.o.f of these joints are respectively $1,2,3$ etc. These numbers are fuzzy i.e., strictly not true but are representative.

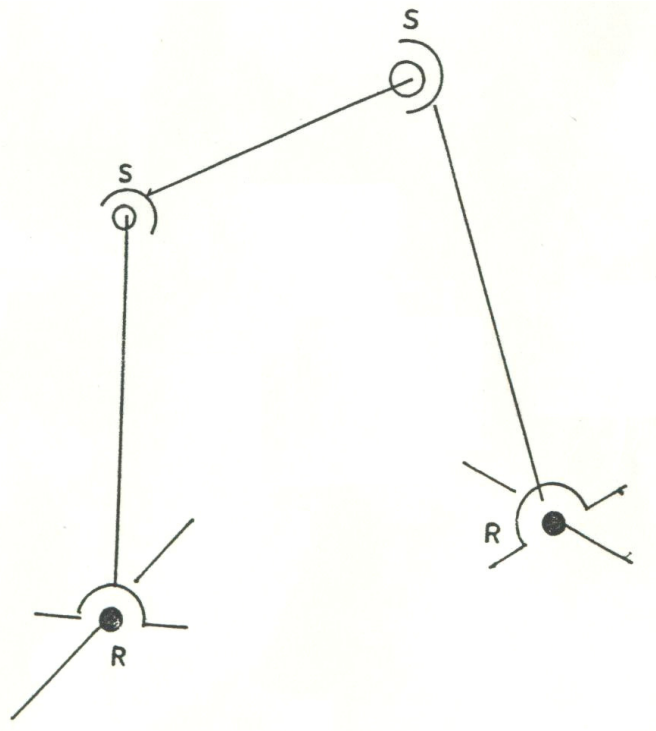

(a) RSSR CHAIN

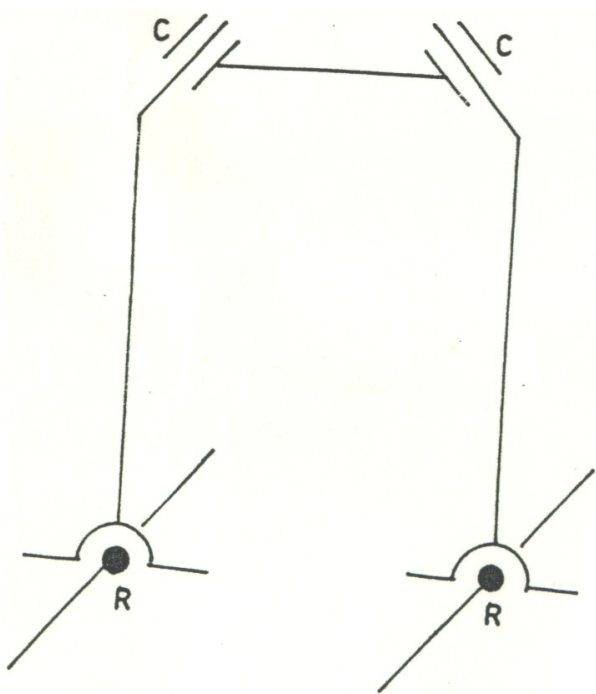

(b) RCCR CHAIN

FIGURE 4

For illustration, let us consider the two chains fig 4 (a\&b), the chain figure4 (a) being RSSR chain its fuzzy vector is

$\begin{array}{llll}0.1 & 0.3 & 0.3 & 0.1\end{array}$

Computing shortest and farthest distances from the respective crisp vectors, one can get fuzzy entropy as 0.25 .

The chain, figure 4(b) is a RCCR chain and its fuzzy vector

$\begin{array}{llll}0.1 & 0.2 & 0.2 & 0.1\end{array}$ 
The above vector leads to a fuzzy entropy of 0.176 . Comparison of fuzzy entropies shows that the entropy is less in the RCCR chain and hence it is more "parallel" in nature.

In case of distinct spatial chains which leads to same fuzzy entropy, obtained in the above manner the entropy may be computed on the basis of adjacency -matrix, the elements of which are the fuzzy numbers. This aspect has already been explained in case of planar chains.

\section{EXPERIMENTAL VERIFICATION}

In order to verify the truth of the results a seven link, 2 d.o.f manipulator is fabricated. Aluminum strips are used as links. All the links are taken of equal size with the specific purpose of obviating the dimensional influence. Length of each binary link is $20 \mathrm{~cm}$ while each ternary link has all the sides equal to $13 \mathrm{~cm}$. Two distinct structures, figure 2 (a) and 3 (b) are considered. Fuzzy logic reveals that the structure figure 3 (b) is more serial than that of the figure 2 (a). Mid point between the joints 3 and 4 of the chain is taken as the end point while for the chain, figure 3(b), mid point of the joints 5-6 is taken as the end point. The end-effector links in both the cases are chosen as they are the maximum floating links i.e., separated by the maximum number of joints from the fixed link (frame). The work spaces for both the chains figure 2 (a) and figure 3 (b) , in that order, are found to be approximately 1000 sq.cm and 1700 sq.cm. The results show that the chain figure 3(a) being more serial has resulted in greater work space proving the validity of the theory presented.

\section{CONCLUSIONS}

1. Fuzzy logic can be used to compare the chains with the same number of links and Degree-of-freedom.

2. Chains containing joints with higher connectivity will tend to be more "serial" and are expected to have more velocity.

3. Chains having the same type and number of joints can be compared on the basis of adjacency fuzzy vectors and entropy. "Greater the entropy indicates less parallelism and more tendency towards serialism.

4. All the distinct multi-degree-of-freedom chains can be rated for parallelism. The chains having more Parallel are more rigid, and will lead to less work space and less positional (joint) errors.

5. Work space aspect is experimentally verified and found in line with the theory presented.

6. It can be generalized that links of a chain with greater entropy are more mobile i.e. the displacements and velocities can be higher.

7. All the possible kinds of distinct multi-degree-of-freedom chains are compared for parallelism using Fuzzy logic and taking the concept of fuzzy entropy by dividing into four different types.

\section{REFERENCES}

1. Hunt, K. H. 1983. "Structural Kinematics of in-parallel actuated robot arms" ASME J. Mechanism, Transmission and Automation in Design,105 (4), PP 705-712. 
2. Pennock, G.R. and D.I. Kassner, 1992. "Kinematic Analysis of a Planar Eight Bar linkage Application to a Platform type Robot", ASME J. Mechanical Design, 144, PP $87-95$.

3. Parenti C.V. and C. Innocenti, 1992. "Forward Displacement analysis of parallel mechanisms: Closed Form solution of PPR3S structures" ASME J. Mechanical Design, PP 144, 68-72.

4. Mechael Chester, 1993. Neural Networks, A tutorial,PTR Prentice Hall,NewJersey1993, PP 119- 130.

5. Uicker J.J. Jr, A. 1975. Raicu, Mechanism and Machine Theory 10 PP 375-383.

6. Chester, M. 1993. Neural Networks: a Tutorial, PTR Prentice Hall, Englewood Cliffs, NJ.

7. Tischler, C.R., A.E. Samuel, K.H. Hunt, 1995. Kinematic chains for robot hands 1. Orderly number synthesis, Mechanism and Machine Theory 35 PP 1193-1215.

8. Ambekar, A.G., V.P. Agrawal, 1987. Canonical numbering of kinematic chains and isomorphism problem, Mincode, Mechanism and Machine Theory 22(5) PP 1-8.

9. Pennestri, E. 1993. Mechanism and Machine Theory (28)721-724. 